\title{
Presence of genes for type III secretion system 2 in Vibrio mimicus strains
}

\author{
Natsumi Okada 1,2, Shigeaki Matsuda', Junko Matsuyama ${ }^{3}$, Kwon-Sam Park ${ }^{4}$, Calvin de los Reyes ${ }^{2}$, Kazuhiro Kogure ${ }^{5}$, \\ Takeshi Honda ${ }^{2}$, Tetsuya lida ${ }^{*}$
}

\begin{abstract}
Background: Vibrios, which include more than 100 species, are ubiquitous in marine and estuarine environments, and several of them e.g. Vibrio cholerae, V. parahaemolyticus, V. vulnificus and V. mimicus, are pathogens for humans. Pathogenic $V$. parahaemolyticus strains possess two sets of genes for type III secretion system (T3SS), T3SS1 and T3SS2. The latter are critical for virulence of the organism and be classified into two distinct phylogroups, T3SS2 $\alpha$ and T3SS2 $\beta$, which are reportedly also found in pathogenic $V$. cholerae non-01/non-O139 serogroup strains. However, whether T3SS2-related genes are present in other Vibrio species remains unclear.

Results: We therefore examined the distribution of the genes for T3SS2 in vibrios other than $V$. parahaemolyticus by using a PCR assay targeting both T3SS2 $\alpha$ and T3SS2 $\beta$ genes. Among the 32 Vibrio species tested in our study, several T3SS2-related genes were detected in three species, V. cholerae, V. mimicus and $V$. hollisae, and most of the essential genes for type III secretion were present in T3SS2-positive $V$. cholerae and V. mimicus strains. Moreover, both $V$. mimicus strains possessing T3SS2 $\alpha$ and T3SS2 $\beta$ were identified. The gene organization of the T3SS2 gene clusters in $V$. mimicus strains was fundamentally similar to that of $V$. parahaemolyticus and $V$. cholerae in both T3SS2 $\alpha$ - and T3SS2 $\beta$-possessing strains.

Conclusions: This study is the first reported evidence of the presence of T3SS2 gene clusters in $V$. mimicus strains. This finding thus provides a new insight into the pathogenicity of the $V$. mimicus species.
\end{abstract}

\section{Background}

The type III secretion system (T3SS) is possessed by gram-negative bacteria, especially those occurring in animal and plant pathogens, e.g. Yersinia, Shigella, Salmonella, Pseudomonas and Escherichia species [1-3]. The T3SS secretes and translocates effector proteins into the cytosol of eukaryotic cells, thus contributing to bacterial virulence against the host [1]. While the T3SS apparatus is well conserved in these bacteria, the specific properties of the effectors which are secreted via T3SS and symptomatic effects caused by the effectors on the host organism vary widely [1].

Vibrios are gram-negative $\gamma$-proteobacteria which are ubiquitous in marine and estuarine environments $[4,5]$. Several of the more than 100 Vibrio species are

\footnotetext{
* Correspondence: iida@biken.osaka-u.ac.jp

'Laboratory of Genomic Research on Pathogenic Bacteria, International Research Center for Infectious Diseases, Research Institute for Microbial Diseases, Osaka University, Osaka, Japan

Full list of author information is available at the end of the article
}

pathogens for fish, shellfish, coral, and mammals [6], and Vibrio parahaemolyticus was the first species in which the presence of T3SS was reported [7].

$V$. parahaemolyticus is a cause of food-borne gastroenteritis in humans, and almost all strains isolated from diarrheal patients produce the thermostable direct hemolysin (TDH) and/or the TDH-related hemolysin (TRH), which are encoded by the $t d h$ and trh genes, respectively [8-10]. V. parahaemolyticus strains, which exhibit the Kanagawa phenomenon (KP), a beta-hemolysis detectable on a special blood agar (Wagatsuma agar) [11], possess two $t d h$ genes, $t d h A$ and $t d h S$, but not the trh gene $[10,12,13]$. In contrast, KP-negative clinical $V$. parahaemolyticus strains possess the trh gene only or both the trh and $t d h$ genes. Genome sequencing of the KP-positive $V$. parahaemolyticus strain RIMD2210633 demonstrated that it possesses two sets of the genes for T3SS on chromosomes 1 and 2 (T3SS1 and T3SS2, respectively) [7]. It has further been demonstrated that T3SS2 is involved in enterotoxicity of the organism, and
Ciomed Central 
is considered to be an important factor in the pathogenicity of diarrheal illness [14]. The T3SS2 genes are located on a pathogenicity island (PAI) known as Vp-PAI on chromosome 2, and the genes were found in KP-positive, but not in KP-negative strains $[7,14,15]$.

After the discovery of the T3SS genes in $V$. parahaemolyticus, other vibrios such as $V$. alginolyticus, $V$. harveyi, $V$. tubiashii and $V$. cholerae were also found to possess the genes for T3SS [14,16-18]. While the T3SSs of $V$. alginolyticus, $V$. harveyi and $V$. tubiashii, are more closely related to T3SS1 of $V$. parahaemolyticus [14], that of $V$. cholerae is similar to T3SS2 of V. parahaemolyticus [17]. In addition, several studies have demonstrated that some $V$. cholerae non-O1/non-O139 serogroup strains, which do not possess the cholera toxin gene, do possess a set of T3SS genes in a PAI (VPI-2) on their chromosome [17,19]. It has further been suggested that the T3SS of non-O1/non-O139 $\mathrm{V}$. cholerae is also involved in the pathogenicity of the bacterium [17].

In our most recently reported study, we used the sequencing and PCR assay of the genomic DNA of the TH3996 strain to detect the presence of a novel PAI (Vp-PAI ${ }_{\mathrm{TH} 3996}$ ) in trh-positive (KP-negative) V. parahaemolyticus strains [20]. The $\mathrm{VP}-\mathrm{PAI}_{\mathrm{TH} 3996}$ was found to contain a set of genes for T3SS, and the T3SS of the TH3996 strain to be essential for the enterotoxicity of this strain [20]. Phylogenetic analysis indicated that the T3SS genes of TH3996 are related to that of RIMD2210633, but belong to distinct lineage, with the former known as T3SS2 $\beta$ and the latter as T3SS2 $\alpha$ [20]. Subsequent studies showed that T3SS $2 \alpha$ and T3SS $2 \beta$ are present in, respectively, KP-positive and trh-positive $V$. parahaemolyticus strains and are also distributed among pathogenic $V$. cholerae non-O1/non-O139 serogroup strains [20].

A previous study examined the distribution of the T3SS2-related genes in Vibrio species, but tested only for the presence of the T3SS2 $\alpha$ genes and in a limited number of strains from each species [14]. In this study, we re-investigated the distribution of the genes for T3SS2 in various Vibrio species and targeted both the T3SS $2 \alpha$ and T3SS2 $\beta$ genes.

\section{Results}

Distribution of the T3SS2-related genes in Vibrio species

To analyze the distribution of the T3SS2-related genes in Vibrio species other than V. parahaemolyticus, PCR assays were performed using oligonucleotide primer pairs (see Additional file 1) which target the T3SS2related genes present in the Vp-PAI, i.e., $v s c N 2$ (encodes the ATPase), $v s c C 2 N 2 R 2 S 2 T 2 U 2, v c r D 2$ (apparatus proteins of T3SS), vopB2D2 (translocons), or vopCLP (effectors) [14,21-24], for 32 Vibrio species. The design of the
PCR primer pairs was based on the gene sequences in strains RIMD2210633 or TH3996, representing T3SS2 $\alpha$ or T3SS2 $\beta$, respectively (see Additional file 1 ). We tested multiple strains of several species in the genus Vibrio which are implicated as pathogenic for humans, that is, V. vulnificus (10 strains), V. fluvialis (12 strains), $V$. furnissii (12 strains), V. hollisae (5 strains), V. cholerae (46 strains) and V. mimicus (15 strains). For other species, one strain of each was tested (see Additional file 2).

The assay demonstrated that, in addition to part of the $V$. cholerae strains, as previously reported, amplicons of the expected size of at least several of the T3SS2 genes were obtained from all of the $V$. hollisae strains and some of the $V$. mimicus strains. However, none of the genes tested in any of the remaining 29 species could be amplified (see Additional file 2).

Among the 46 non-O1/non-O139 V. cholerae strains isolated from patients (28 strains) or environments (18 strains), we obtained the amplicons of at least one gene encoding the apparatus protein of the T3SS2 $\alpha$ genes from 10 strains (see below). In two $V$. cholerae strains, which constitute the PCR products of T3SS $2 \beta$ genes, at least six genes for the apparatus and two genes for the translocons could be amplified (see Additional file 2). We therefore concluded that the aforementioned $10 \mathrm{~V}$. cholerae strains were T3SS2 $\alpha$-positive and the two were T3SS2 $\beta$-positive. Of these 12 T3SS2-positive strains, only one, the $V$. cholerae strain RIMD2214415, which possesses T3SS $2 \alpha$ genes, was isolated from the environment. Therefore, as far as we could determine in this study, T3SS2 genes of $V$. cholerae tend to be found in clinical strains rather than in environmental isolates.

In all of the five $V$. hollisae strains tested, the amplicons for three genes of T3SS $2 \alpha, v s c N 2, v s c R 2$ and $v s c T 2$, were obtained with the PCR assay, but no other T3SS $2 \alpha$ genes or any T3SS2 $\beta$ genes could be amplified. The PCR products for $v s c N 2 R 2 T 2$ could be partially sequenced, which confirmed that the amplicons that could be obtained are more closely related to the T3SS $2 \alpha$ than to the T3SS2 $\beta$ genes (data not shown).

The PCR products of the genes for T3SS2 were detected in nine of 15 clinical or environmental $V$. mimicus strains. The genes encoding the apparatus proteins of T3SS2, vscN2C2R2T2U2 and $v c r D 2$, were amplified by PCR in all the T3SS2-positive $V$. mimicus strains, although the amplicons for the genes encoding effector proteins, i.e., $\operatorname{vop} C L P$, could not be obtained in a few of these strains (see Additional file 2). Of the nine T3SS2positive strains, at least six genes for the apparatus proteins and two genes for the translocons of T3SS2 $\alpha$ genes could be amplified from eight strains, while PCR amplification led to the detection in a $V$. mimicus strain of the amplicons of the T3SS2 $\beta$ genes, i.e., six genes 
encoding the apparatus proteins $v s c N 2 C 2 R 2 T 2 U 2$ and $v c r D 2$, two genes encoding the translocons vopB2D2, and two genes for the regulators $v \operatorname{tr} A B$. In the other six $V$. mimicus strains, no amplicons of the genes for either type of T3SS2 could be obtained (see Additional file 2). Of the nine T3SS2-positive $V$. mimicus strains, eight were therefore identified as T3SS $2 \alpha$-positive, and one as T3SS2 $\beta$-positive. These findings suggest that, in addition to their distribution in $V$. parahaemolyticus and $V$. cholerae strains, the genes for T3SS2 are found in $V$. hollisae and $V$. mimicus strains, although only three T3SS2 genes were detected in $V$. hollisae strains.

\section{Distribution of $t d h$ or trh in T3SS2-positive $V$. cholerae and $V$. mimicus strains}

In $V$. parahaemolyticus, the strains which possess the T3SS2 gene cluster also possess the $t d h$ and/or trh genes $[7,20]$. To examine whether the $t d h$ or $t r h$ genes also coexist in T3SS2-possessing $V$. cholerae and $V$. mimicus strains, we performed a PCR assay using the pair of primers (see Additional file 1) which target the $t d h$ or trh genes of $V$. parahaemolyticus RIMD2210633 or TH3996 strains, respectively. Among the T3SS2-possessing $V$. mimicus strains, the $t d h$ gene could be detected in all T3SS2 $\alpha$-positive strains, although no amplicons of the trh gene could be obtained in $V$. mimicus strains, while neither of the amplicons could be obtained in the T3SS2positive $V$. cholerae strains. To analyze the distribution of the $t d h$ or $t r h$ genes in 12 T3SS2-positive $V$. cholerae strains, we performed an additional PCR assay using the primer set (see Additional file 1) targeting the region between A33_1702 and the downstream region of the $V$. cholerae AM-19226 strain which is homologous with $t d h$ of $V$. parahaemolyticus. PCR products could be obtained for both the T3SS $2 \alpha$ - and T3SS2 $\beta$-positive $V$. cholerae strains, except for the RIMD2214321 (T3SS2 $\alpha$-possessing) strain (data not shown). These results suggest that the $t d h$ gene may be related to the presence of the T3SS2 gene cluster in $V$. cholerae and $V$. mimicus strains.

\section{Gene organization of the T3SS2 gene cluster in $V$. mimicus}

The results presented here demonstrated that some $V$. mimicus strains possess the genes for T3SS $2 \alpha$ or T3SS2 $\beta$. Since the gene organizations of the T3SS2 gene cluster in the organism were completely unknown, we attempted to analyze the gene organization of the T3SS2 region in $V$. mimicus strains. To this end, we performed PCR scanning against the genomic DNA of T3SS2-positive $V$. mimicus strains RIMD2218080 (T3SS2 $\alpha$ ) and RIMD2218067 (T3SS2 $\beta$ ) by using six PCR primer pairs for each strain (see Additional file 3 ).

In the T3SS2 $\alpha$-positive strain RIMD2218080, PCR products of the expected size were detected for all primer pairs (data not shown), thus suggesting that the gene organization of the T3SS2 gene cluster in strain RIMD2218080 is similar to that of the T3SS2 $\alpha$ gene cluster in $V$. parahaemolyticus strain RIMD2210633. In the T3SS2 $\beta$-positive strain RIMD2218067, amplicons of the expected size were obtained for five primer pairs, although the size of the product obtained for a primer pair between the vopD2 and $v o p C$ genes was notably larger, by approximately $5 \mathrm{~kb}$, than that of the region of the $V$. parahaemolyticus TH3996 (data not shown). However, the PCR product which was amplified between vopD2 and vopP was the same size as that of the $V$. parahaemolyticus TH3996 strain. This suggested that the gene organization of T3SS2 of $V$. mimicus RIMD2218067 is similar to that of T3SS2 $\beta$ of $V$. parahaemolyticus, while a genetic element of approximately $5 \mathrm{~kb}$, which could not be detected in the region of $V$. parahaemolyticus strain TH3996, may exist in the region between the vopP and vopC genes in $V$. mimicus strain RIMD2218067.

These findings suggest that the gene organization of the T3SS 2 gene clusters, both T3SS $2 \alpha$ and T3SS $2 \beta$, in $V$. mimicus strains are basically similar to those of the $V$. parahaemolyticus and $V$. cholerae strains.

\section{Phylogenetic analysis of the T3SS2-related genes in $V$. mimicus}

Next, we analyzed the phylogeny of the T3SS2 genes identified in $V$. mimicus strains. The purified amplicons of the genes for $v s c N 2 R 2 T 2$ in the T3SS2-positive $V$. mimicus strains were sequenced and the nucleotide sequences thus obtained were used for phylogenetic analysis. In addition, we used the nucleotide sequences of the three T3SS2 genes of the two $V$. parahaemolyticus strains RIMD2210633 and TH3996, and the four $V$. cholerae strains, AM-19226, 1587 and 623-39, as well as V51, identified to date. Phylogenetic trees for each of the genes were constructed with the Neighbor-Joining (NJ) method.

The analysis demonstrated that the PCR products of the T3SS2 genes in $V$. mimicus strains RIMD2218022, 2218042, 2218069, 2218070, 2218080, 2218081, 2218082 and 2218083 belong to the cluster containing the T3SS2 $\alpha$ genes of $V$. parahaemolyticus strain RIMD2210633 and that of $V$. cholerae strains AM19226 and V51 (Figure 1). In contrast, the amplicons obtained from the T3SS2 genes in the $V$. mimicus strain RIMD2218067 were found to be closely related to the T3SS2 $\beta$ genes in the $V$. parahaemolyticus TH3996 strain and $V$. cholerae strains 1587 and 623-39 (Figure 1). These findings confirmed that, similar to the findings for $V$. parahaemolyticus and $V$. cholerae strains, the T3SS2 of $V$. mimicus strains could be classified into two phylogroups, T3SS $2 \alpha$ and T3SS $2 \beta$. 


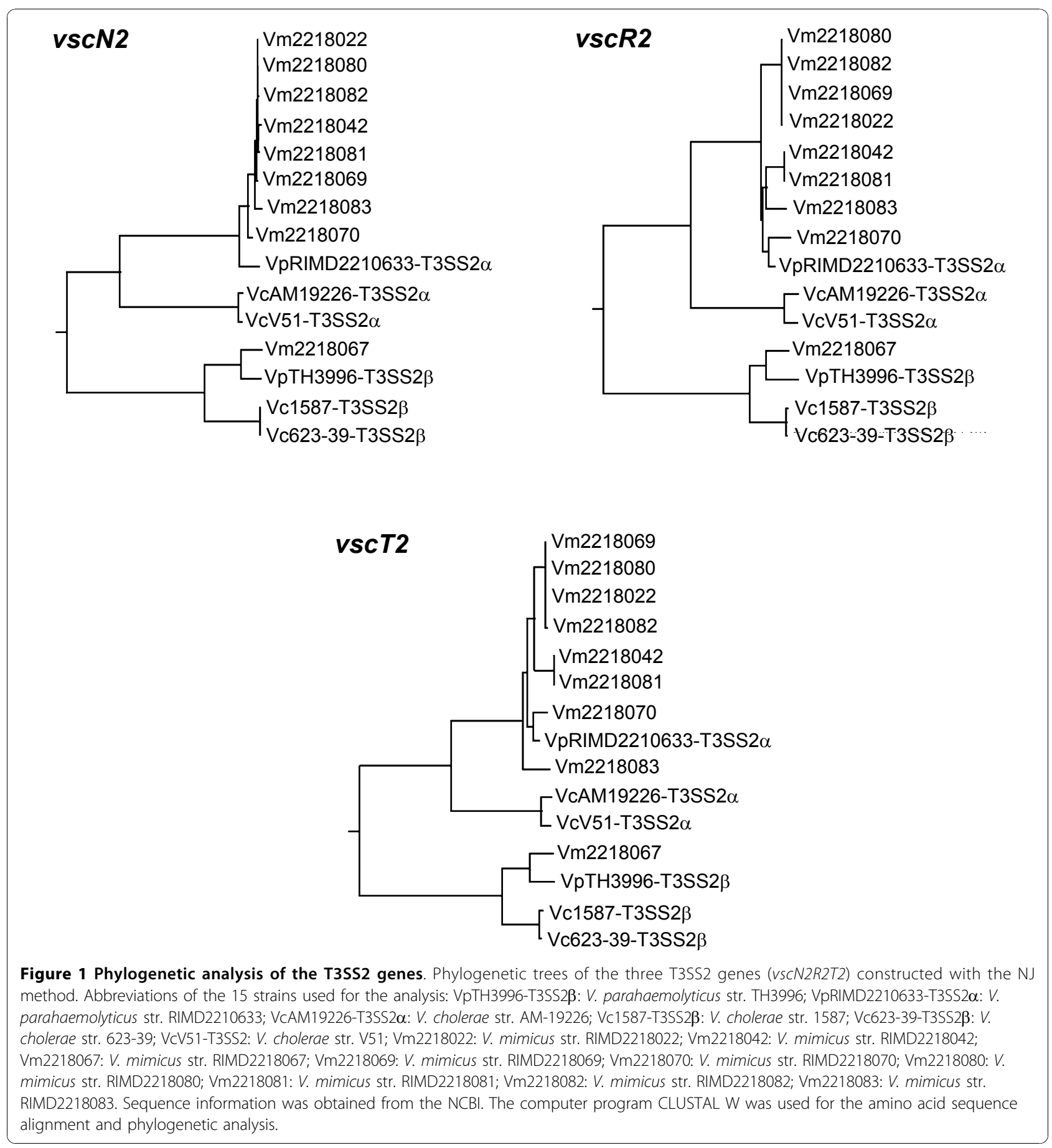

\section{Presence and absence of the genes in VPI-2 and Vp-PAI}

Both the T3SS2 gene cluster of $V$. parahaemolyticus and the T3SS gene cluster of $V$. cholerae can be found on PAIs $[7,19,20]$. In $V$. cholerae non-O1/non-O139 strains AM-19226, NRT36S, V51, 1587 and 623-39, the PAI (VPI-2) contains the genes for sialic acid metabolism in addition to the genes for T3SS (Figure 2) [19]. In $V$. parahaemolyticus strains RIMD2210633 and TH3996, on the other hand, the homologues for sialic acid metabolism were not found in the Vp-PAI (Figure 2). The gene compositions of the PAI cassettes in $V$. parahaemolyticus and $V$. cholerae, except for the T3SS gene cluster, were thus clearly distinct. To compare the gene organization of the PAI of $V$. mimicus with that of the PAIs of $V$. parahaemolyticus and $V$. cholerae, we used additional PCR assays to determine the presence or 


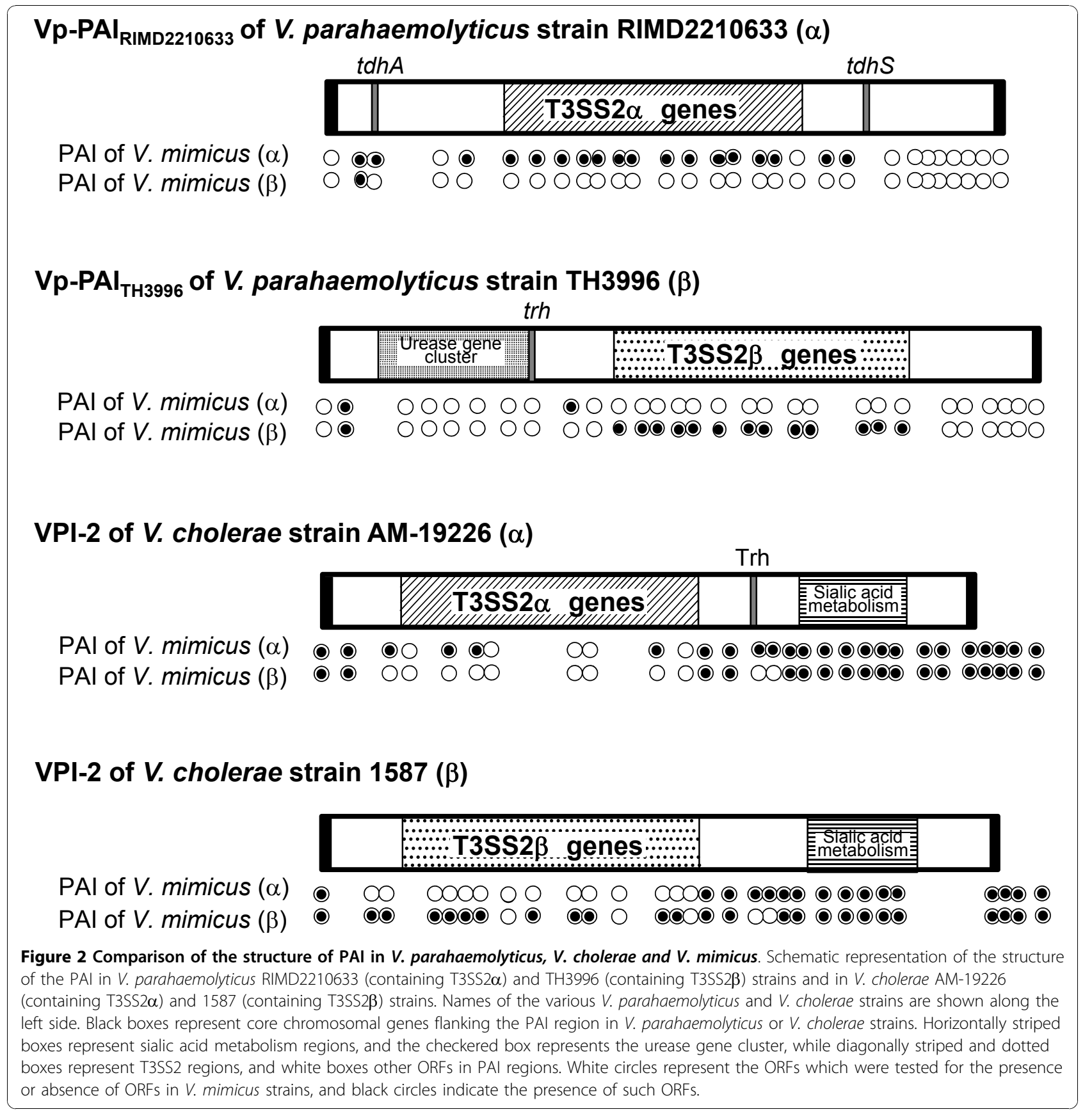

absence of open reading frames (ORFs), which occur only in the Vp-PAI of $V$. parahaemolyticus or the VPI-2 of $V$. cholerae, in T3SS2-positive $V$. mimicus strains. The ORFs on the PAIs of $V$. parahaemolyticus and $V$. cholerae strains, except for the T3SS2 genes, could be amplified with the primer sets that were designed by using the ORF sequences on the Vp-PAI in V. parahaemolyticus strains RIMD2210633 and TH3996 and those on VPI-2 in $V$. cholerae strains AM-19226 and 1587 as templates against the genomic DNA of nine $V$. mimicus
T3SS2-positive strains (see Additional file 4, 5, 6, 7). Some of the ORFs on the Vp-PAI of $V$. parahaemolyticus strains, could be amplified in the $V$. mimicus strains tested, but most could not (see Additional file 4, 5, 6, 7, Figure 2). In contrast, most of the non-T3SS ORFs on VPI-2 of $V$. cholerae could be amplified in the T3SSpositive, but not in the T3SS2-negative $V$. mimicus strains (data not shown).

These findings suggest that the composition of the $V$. mimicus PAIs containing the T3SS genes, if present, 
may be more closely related to that of $V$. cholerae VPI-2 than of $V$. parahaemolyticus Vp-PAI (Figure 2).

\section{Cytotoxicity assay of mutant strains}

Previous studies have demonstrated that T3SS2s of $V$. parahaemolyticus RIMD2210633 and TH3996 as well as $V$. cholerae AM-19226 contribute to the pathogenicity of these organisms $[14,17,20,22-24]$. To determine the possible contribution of T3SS2 to the pathogenicity of $V$. mimicus strains, we compared the cytotoxicity of the wild-type and mutant strains for cultured cell lines. T3SS-deficient mutants were constructed by disruption of the homologue of the $v s c N 2$ gene, which encodes an ATPase of T3SS2, in $V$. mimicus RIMD2218042 ( $\alpha$ type) and RIMD2218067 ( $\beta$ type) strains. To confirm the deletion of the $v s c N 2$ gene, PCR amplification using oligonucleotide primer pairs was performed (see Additional file 1 and 8). The growth of the mutant strains in LB medium $(1 \% \mathrm{NaCl})$ was indistinguishable from that of the parental strains (data not shown). Both $V$. mimicus RIMD2218042 and RIMD2218067 strains were cytotoxic for Caco- 2 cells at $3 \mathrm{~h}$ post-infection. The cytotoxicity of both the T3SS $2 \alpha$ - and T3SS $2 \beta$-deficient mutant strains tended to decrease, but there were no significant differences between T3SS $2 \alpha$ - and T3SS $2 \beta$-deficient mutant strains and their parental strains (see Additional file 9).

\section{Discussion}

A recent study of ours demonstrated that two lines of distinct lineage of the T3SS2 gene cluster, T3SS2 $\alpha$ and T3SS2 $\beta$, are present in the KP-positive and trh-positive $V$. parahaemolyticus strains, respectively [20]. Although a previously reported study using dot blot analysis could not detect the genes for T3SS2 in 16 Vibrio species, the probes and PCR primers used in previous studies were designed based on the sequence information of the T3SS $2 \alpha$ genes in $V$. parahaemolyticus strain RIMD2210633 [7,14]. Since the T3SS2 $\beta$ genes cannot be detected by either PCR amplifications or comparative genomic hybridization analysis targeting the T3SS $2 \alpha$ genes $[7,15]$, we re-investigated the distribution of the T3SS 2 genes, both T3SS $2 \alpha$ and T3SS $2 \beta$, in Vibrio species.

To examine the distribution of the genes for T3SS2 in vibrios other than $V$. parahaemolyticus, we performed a PCR assay using PCR primer pairs targeting both the T3SS $2 \alpha$ and T3SS $2 \beta$ genes. Of the 32 Vibrio species tested, the T3SS2-related genes were detected in three species, $V$. cholerae, which was previously reported, as well as $V$. hollisae and $V$. mimicus.

In $V$. hollisae strains, only three genes for $\mathrm{T} 3 \mathrm{SS} 2 \alpha$, $v s c N 2$, vscR2, and $v s c T 2$, were detected. Nevertheless, the fact that the PCR reactions for these three genes were positive in all the five $V$. hollisae strains tested is intriguing. We speculate that the other genes for T3SS2 $\alpha$ might be absent in these particular $V$. hollisae strains, or that the sequences of the other genes included variations that would make PCR amplification with the primer pairs used in this assay difficult. These possibilities should be examined in the future by more detailed genetic analyses, e.g. sequencing of the region flanking the T3SS2-related genes.

A previous study showed that the T3SS2-related genes are present in $V$. mimicus strains [25]. In our study, the PCR assay also demonstrated the presence of the T3SS2 genes in $V$. mimicus strains. Of the $15 \mathrm{~V}$. mimicus strains tested in this study, 12 strains were isolated from patients and three from the environment, with all T3SS2-positive strains isolated from patients. There were eight T3SS $2 \alpha$-positive and one T3SS $2 \beta$-positive strain among the T3SS2-positive $V$. mimicus strains. The gene organization of the T3SS2 gene cluster in the $V$. mimicus strains containing T3SS $2 \alpha$ or T3SS $2 \beta$, was basically similar to that of the $V$. parahaemolyticus and $V$. cholerae strains. Ours is thus the first study to demonstrate that the two distinct types of T3SS2 gene clusters, T3SS $2 \alpha$ and T3SS2 $\beta$, are present not only in $V$. parahaemolyticus and $V$. cholerae but also in $V$. mimicus strains. Furthermore, we could show that the structures of the $V$. mimicus PAIs containing the T3SS genes may be more closely related to those of $V$. cholerae VPI2 than of $V$. parahaemolyticus Vp-PAI (Figure 2). In contrast, the ORFs in VPI-2 were not detected in any of the T3SS-negative $V$. mimicus strains. This implies, therefore, that the similar PAI cassettes containing the T3SS2 gene cluster were acquired through horizontal gene transfer in $V$. cholerae and $V$. mimicus (Figure 3 ).

The PCR primer pairs used in this study were found to be useful for detecting as well as distinguishing the genes for T3SS $2 \alpha$ and T3SS2 $\beta$ in Vibrio species. In particular, the PCR assays targeting the three genes, $v s c N 2$, $\nu s c R 2$ and $v s c T 2$, produced stable and reliable results for detection of T3SS2-related genes. We therefore consider that, for determining the presence or absence of these genes, PCR amplification using the primer pairs for the $\nu s c N 2 R 2 T 2$ genes of T3SS $2 \alpha$ or T3SS $2 \beta$ is effective and rapid. Although only a limited number of strains of the non-human pathogenic Vibrio species was examined in this study, more extensive studies of those species using more strains may well reveal the presence of the T3SS2 genes in vibrios other than the ones reported here.

Previous studies showed that the T3SSs of $V$. parahaemolyticus and $V$. cholerae contribute to their pathogenicity for humans $[14,17,20,22-24]$. In $V$. mimicus, a bacterium which is known to be a causative agent of gastroenteritis in humans, the hemolysin was previously reported as a major virulence factor [26]. To assess the 


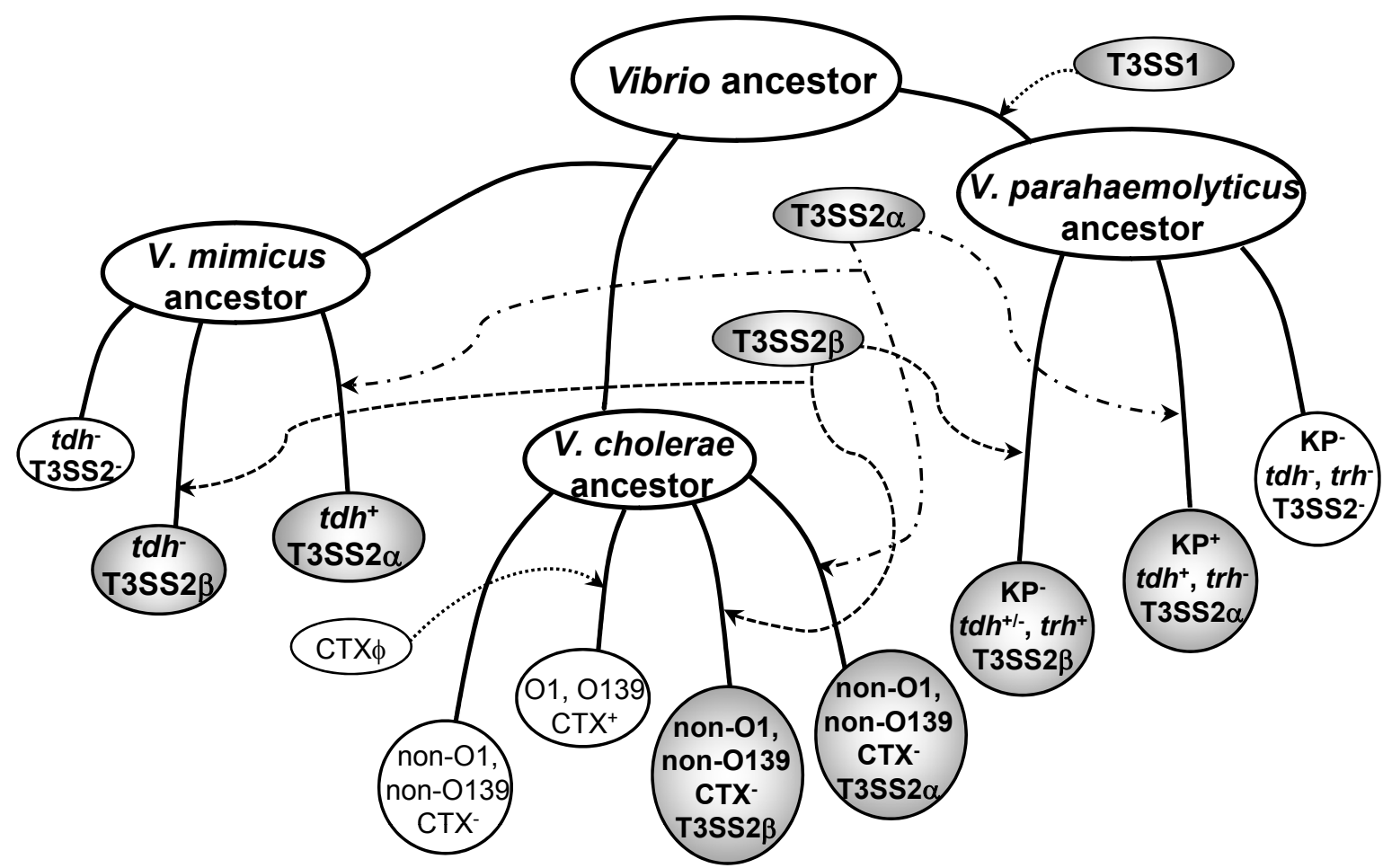

Figure 3 Schematic representation of the hypothetical evolutionary acquisition of the T3SS-related gene cluster in $V$. parahaemolyticus, $\boldsymbol{V}$. cholerae and $\boldsymbol{V}$. mimicus. Lineage is based on the presence of each of the determinants, for example, tdh, trh, CTX and T3SS2. The shaded ellipses show the T3SS-related gene clusters, bold lines represent the evolutionary process, and circles represent the strains of $V$. parahaemolyticus, V. cholerae and V. mimicus, while shaded circles indicate that the strains possess T3SS $\alpha$ or T3SS $\beta$. Broken lines indicate that the T3SS gene clusters or CTX have been acquired by horizontal gene transfer while the organisms were evolving.

function of T3SS of $V$. mimicus in pathogenicity in our study, we evaluated the cytotoxicity of $V$. mimicus for Caco-2 cells because $V$. parahaemolyticus had a T3SS2dependent cytotoxic effect on Caco-2 cells [22]. The results indicated that both T3SS $2 \alpha$-possessing and T3SS2 $\beta$-possessing $V$. mimicus strains showed the cytotoxic activity on Caco-2 cells in this assay. Although we could not detect statistically significant differences between T3SS-deficient mutants and parental strains, there was a tendency for the cytotoxicity of T3SS-deficient mutants to diminish than that of the parental mutants. A previous report showed that the deletion of the hemolysin gene in $V$. mimicus significantly reduced fluid accumulation in rabbit ileal loop tests, but the mutant partially retained this action, which suggests that, besides the hemolysin, $V$. mimicus may contain an additional virulence determinant(s) [26]. It is therefore possible that T3SS is a candidate for the previously unidentified virulence determinant in pathogenic $V$. mimicus strains for humans. The observed ambiguous differences in cytotoxicity between the mutants and the parental strains may be due to insufficient expression of T3SS of $V$. mimicus under the culturing conditions used in this study, because it is still unclear what the optimal conditions are for inducing T3SS of $V$. mimicus. This possibility needs to be examined in future studies.

\section{Conclusions}

This study demonstrated the presence of the gene cluster for T3SS $2 \alpha$ or T3SS2 $\beta$ in $V$. mimicus, a bacterium which is known to be a causative agent of gastroenteritis in humans. Since it was reported that the T3SSs of $V$. parahaemolyticus and $V$. cholerae contribute to their pathogenicity for humans, the T3SS in $V$. mimicus identified in this study also might be a candidate virulence factor of this organism for humans. This possibility needs to be examined in future studies.

\section{Methods}

Bacterial strains and growth conditions

All the Vibrio species strains were obtained from the Pathogenic Microbes Repository Unit, International Research Center for Infectious Diseases, Research Institute for Microbial Diseases, Osaka University. The culture temperatures were $15^{\circ} \mathrm{C}$ for $V$. logei and $V$. salmonicida and $10^{\circ} \mathrm{C}$ for $V$. wondanis, while all other bacteria were cultured at $25^{\circ} \mathrm{C}$. The bacteria were grown with shaking in Luria-Bertani (LB) broth (tryptone, 1\%; 
yeast extract, $0.5 \%$ ) with $3 \% \mathrm{NaCl}$ for $V$. parahemolyticus and in Difco marine broth 2216 for V. nigripulchritudo, $V$. pectenicida and $V$. halioticoli. Other bacteria were grown in $\mathrm{LB}$ broth with $1 \% \mathrm{NaCl}$.

\section{Oligonucleotide primers and PCR conditions}

Additional file 1 shows the oligonucleotide primers used in this study. Chromosomal DNA from Vibrio species strains was extracted for PCR as previously described [20]. For detection of the presence of the T3SS2 genes in related Vibrio species, PCR using the EX-PCR Kit (Takara Shuzo, Kyoto, Japan) was performed. The PCR conditions were as follows: after initial denaturation at $94^{\circ} \mathrm{C}$ for $3 \mathrm{~min}$, a cycle of $94^{\circ} \mathrm{C}$ for $30 \mathrm{~s}, 55^{\circ} \mathrm{C}$ for $30 \mathrm{~s}$, and $72^{\circ} \mathrm{C}$ for $30 \mathrm{~s}, 45 \mathrm{~s}, 1 \mathrm{~min}$ or $1.5 \mathrm{~min}$ was repeated 30 times. PCR scanning of the $V$. mimicus was performed using genomic DNA as a template and with a long accurate (LA) PCR kit (Takara Shuzo) as previously described [20]. Custom-synthesized oligonucleotides for the PCR were purchased from GeneDesign (Osaka, Japan).

\section{DNA sequencing and informatic analysis}

To sequence the DNA fragments amplified by PCR, the fragments were purified with the PCR Gel Extraction Kit (QIAGEN, Valencia, CA) according to the manufacturer's protocol. DNA sequencing was performed with the ABI PRISM 3130 (Applied Biosystems, Foster City, $\mathrm{CA}$ ) and the BigDye v3.1 cycle sequencing kit (Applied Biosystems). The Genetyx sequence analysis program (Software Development, Tokyo, Japan) was used for computer analysis of DNA sequences. Homology searches against deposited sequences were performed with the aid of data from the National Center for Biotechnology Information (NCBI) using the BLAST network service http://www.ncbi.nlm.nih.gov and the BLAST service at the Genome Information Research Center http://genome.naist.jp/bacteria/vpara/. Sequence information was obtained from the NCBI. The computer program CLUSTAL W was used for the nucleotide sequence alignment and phylogenetic analysis.

\section{Construction of vscN2 deletion mutant strains of $V$. mimicus}

A four-primer PCR technique was used to engineer an in-frame deletion mutation as described previously [14]. Briefly, the upstream and downstream sequences of $\nu s c N 2$ of T3SS $2 \alpha$ or T3SS $2 \beta$ were amplified using the pairs listed in Additional file 1. The two fragments, amplified with primers 1 and 3, and 2 and 4, respectively, were used as templates for a second PCR using primers 1 and 4, which generated a PCR product containing the desired deletion. The amplified fragments were then sequenced and subcloned into an R6K-ori suicide vector pYAK1 and transformed into E. coli SM10גpir.

\section{Cytotoxicity assays}

For cytotoxicity assays, eukaryotic cells were seeded at $3 \times$ $10^{4}$ cells well $^{-1}$ in 96 -well plates and cultured for $48 \mathrm{~h}$ to confluency. The cells were co-cultured with PBS-washed bacteria at a multiplicity of infection (moi) of 10 for 2- $6 \mathrm{~h}$. The release of lactate dehydrogenase (LDH) into the medium was quantified by using a CytoTox96 non-radioactive cytotoxicity kit (Promega) according to the manufacturer's instructions. The LDH release (per cent cytotoxicity) was calculated with the following equation: [optical density at $492 \mathrm{~nm}\left(\mathrm{OD}_{492}\right)$ of experimental release - $\mathrm{OD}_{492}$ of spontaneous release]/(OD 492 of maximum release - $\mathrm{OD}_{492}$ of spontaneous release) $\times 100$. Spontaneous release is defined as the amount of LDH released from the cytoplasm of uninfected cells, and maximum release as the total amount of LDH released after the complete lysis of uninfected cells.

\section{Statistical analysis}

Statistical significance was determined with the $t$ test. A $P$ value of $<0.05$ was considered statistically significant.

\section{Nucleotide sequence accession numbers}

The nucleotide sequence data reported in this paper will appear in the DDBJ, EMBL, and GenBank nucleotide sequence database under accession numbers AB560976, AB560977, AB560978, AB560979, AB560980, AB560981, AB5609782, AB560983, AB560984, AB560985, AB560986, AB560987, AB560988, AB560989, AB560990, AB560991, AB560992, AB560993, AB560994, AB560995, AB560996, AB560997, AB560998, AB560999, AB561000, AB561001, and AB561002.

\section{Additional material}

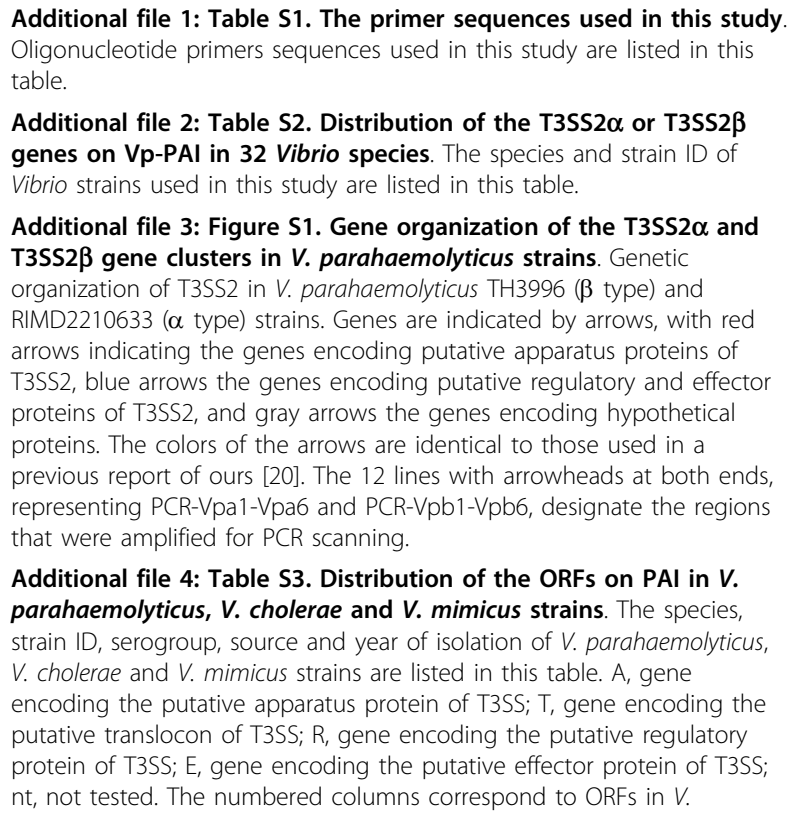


parahaemolyticus RIMD2210633 strain; 1, VPA1309; 2, VPA1312; 3, VPA1314 (tdh gene); 4, VPA1373; 5, VPA1376; 6, VPA1380; 7, VPA1387; 8, VPA1388; 9, VPA1393; 10, VPA1394; 11, VPA1395; 12, VPA1396; 13, VPA1397.

Additional file 5: Table S4. Distribution of the ORFs on PAI in V. parahaemolyticus, $V$. cholerae and $V$. mimicus strains. The species, strain ID, serogroup, source and year of isolation of $V$. parahaemolyticus, $V$. cholerae and $V$. mimicus strains are listed in this table. A, gene encoding the putative apparatus protein of T3SS; T, gene encoding the putative translocon of T3SS; R, gene encoding the putative regulatory protein of T3SS; E, gene encoding the putative effector protein of T3SS; $n t$, not tested. The numbered columns correspond to ORFs in $V$. parahaemolyticus TH3996 strain; 1, orf-1; 2, RPI08 (ureG); 3, RPI15 (nikA); 4, RPI17 (nikC); 5, RPI20 (ureR); 6, RPI23 (ospB); 7, RPI27; 8, RPI80; 9, RPI82; 10, RPI85; 11, RPI87; 12, RPI89; 13, RPI92; 14, orf + 1.

Additional file 6: Table S5. Distribution of the ORFs on PAI in $V$. parahaemolyticus, $V$. cholerae and $V$. mimicus strains. The species, strain ID, serogroup, source and year of isolation of $V$. parahaemolyticus, $V$. cholerae and $V$. mimicus strains are listed in this table. A, gene encoding the putative apparatus protein of T3SS; T, gene encoding the putative translocon of T3SS; R, gene encoding the putative regulatory protein of T3SS; E, gene encoding the putative effector protein of T3SS; nt, not tested. The numbered columns correspond to ORFs in V. cholerae AM-19226 strain; 1, A33_1654; 2, A33_1655; 3, A33_1657; 4, A33_1659; 5, A33_1661; 6, A33_1663; 7, A33_1697; 8, A33_1700; 9, A33_1703; 10, A33_1704; 11, A33_1706; 12, A33_1713; 13, A33_1715; 14, A33_1719; 15, A33_1722; 16, A33_1724; 17, A33_1726; 18, A33_1728.

Additional file 7: Table S6. Distribution of the ORFs on PAI in $V$. parahaemolyticus, $V$. cholerae and $V$. mimicus strains. The species, strain ID, serogroup, source and year of isolation of $V$. parahaemolyticus, $V$. cholerae and $V$. mimicus strains are listed in this table. A, gene encoding the putative apparatus protein of T3SS; T, gene encoding the putative translocon of T3SS; R, gene encoding the putative regulatory protein of T3SS; E, gene encoding the putative effector protein of T3SS; $\mathrm{nt}$, not tested. The numbered columns correspond to ORFs in $V$. cholerae 1587 strain; 1, A55_1978; 2, A55_1980; 3, A55_1981; 4, A55_1982; 5, A55_1983; 6, A55_1984; 7, A55_1988; 8, A55_1989; 9, A55_B0297; 10, A55_B0300; 11, A55_2005; 12, A55_2008; 13, A55_2011; 14, A55_2013; 15, A55_2016; 16, A55_2018; 17, A55_2021; 18, A55_2023; 19, A55_2027; 20, A55_2030; 21, A55_2031.

Additional file 8: Figure S2. PCR amplification of the vscN2 deletion mutant V. mimicus strains. Parental strains (ca. 1200 bp), T3SS-deficient mutant strains (ca. 600bp). The size of the products of the mutant strains was notably smaller, by approximately $600 \mathrm{bp}$, than that of parental strains, including that the mutant strains of vscN2 genes of $\mathrm{V}$. mimicus were constructed. 1, V. mimicus RIMD2218042 (T3SS2 $\alpha$-possessing) strain; 2, V. mimicus RIMD2218042 $\Delta$ vsCN2 (T3SS2 $\alpha$-deficient mutant) strain; $3, V$. mimicus RIMD2218067 (T3SS2 $\beta$-possessing) strain; 4, V. mimicus RIMD2218067 $\triangle v S C N 2$ (T3SS2 $\beta$-deficient mutant) strain.

\section{Additional file 9: Figure S3. Cytotoxicity induced by V. mimicus} against Caco-2 cells. Caco-2 cells were infected with bacteria at an moi of 10. After infection, cytotoxicity was assayed by measuring total cellular $\mathrm{LDH}$ release into the cellular supernatant. The amount of $L D H$ released by Caco-2 cells was measured $3 \mathrm{~h}$ after infection with RIMD2218042 (T3SS2 $\alpha$-possessing) or RIMD2218042 $\Delta$ vsCN2 (T3SS2 $\alpha$-deficient mutant) or RIMD2218067 (T3SS2 $\beta$-possessing) or RIMD2218067 $\Delta v s C N 2$ (T3SS2 $\beta$ deficient mutant) strains.

\section{Acknowledgements}

This work was supported by Grants-in-Aid for Scientific Research on Priority Areas and for Scientific Research from the Ministry of Education, Culture, Sports, Science and Technology of Japan.

\section{Author details}

'Laboratory of Genomic Research on Pathogenic Bacteria, International Research Center for Infectious Diseases, Research Institute for Microbial Diseases, Osaka University, Osaka, Japan. ${ }^{2}$ Department of Bacterial Infections,
Research Institute for Microbial Diseases, Osaka University, Osaka, Japan. ${ }^{3}$ Pathogenic Microbes Repository Unit, International Research Center for Infectious Diseases, Research Institute for Microbial Diseases, Osaka University, Osaka, Japan. ${ }^{4}$ Department of Food Science and Technology, College of Ocean Science and Technology, Kunsan National University, Kunsan, Korea. ${ }^{5}$ Marine Ecosystem Dynamics, Atmosphere and Ocean Research Institute, the University of Tokyo, Tokyo, Japan.

\section{Authors' contributions}

NO designed the study, performed most experiments, interpreted the data and drafted the manuscript. SM shared a part of PCR work, sequencing and cytotoxicity assay, helped to design the study and draft the manuscript. JM and KK isolated and collected Vibrio strains used in the work. KSP and CR assisted study design and data interpretation. TH and TI coordinated the work and drafted the manuscript. All authors read and approved the manuscript.

Received: 9 June 2010 Accepted: 29 November 2010 Published: 29 November 2010

\section{References}

1. Hueck CJ: Type III protein secretion systems in bacterial pathogens of animals and plants. Microbiol Mol Biol Rev 1998, 62:379-433.

2. Jarvis KG, Girón JA, Jerse AE, McDaniel TK, Donnenberg MS, Kaper JB: Enteropathogenic Escherichia coli contains a putative type III secretion system necessary for the export of proteins involved in attaching and effacing lesion formation. Proc Natl Acad Sci USA 1995, 92:7996-8000.

3. Perry RD, Fetherston JD: Yersinia pestis-etiologic agent of plague. Clin Microbiol Rev 1997, 10:35-66.

4. Farmer JJ III, Hickman-Brenner FW: The genera Vibrio and Photobacterium. In The prokaryotes. A handbook on the biology of bacteria: ecophysiology, isolation, identification, and application.. 2 edition. Edited by: Balows A, Trüper HG, Dworkin M, Harder W, Schleifer KH. Berlin: Springer-Verlag KG; 1992:2952-3011.

5. Thompson FL, lida T, Swings J: Biodiversity of vibrios. Microbiol Mol Biol Rev 2004, 68:403-431.

6. Rosenberg E, Ben-Haim Y: Microbial diseases of corals and global warming. Environ Microbiol 2002, 4:318-326.

7. Makino K, Oshima K, Kurokawa K, Yokoyama K, Uda T, Tagomori K, lijima Y, Najima M, Nakano M, Yamashita A, Kubota Y, Kimura S, Yasunaga T, Honda T, Shinagawa H, Hattori M, lida T: Genome sequence of Vibrio parahaemolyticus: a pathogenic mechanism distinct from that of $V$ cholerae. Lancet 2003, 361:743-749.

8. Blake PA, Weaver RE, Hollis DG: Diseases of humans (other than cholera) caused by vibrios. Annu Rev Microbiol 1980, 34:341-367.

9. Honda T, lida T: The pathogenicity of Vibrio parahaemolyticus and the role of the thermostable direct haemolysin and related haemolysin. Rev Med Microbiol 1993, 4:106-113.

10. Nishibuchi M, Kaper JB: Thermostable direct hemolysin gene of Vibrio parahaemolyticus: a virulence gene acquired by a marine bacterium. Infect Immun 1995, 63:2093-2099.

11. Sakazaki R, Tamura K, Kato T, Obara Y, Yamai S: Studies on the enteropathogenic, facultatively halophilic bacterium, Vibrio parahaemolyticus. 3. Enteropathogenicity. Jpn J Med Sci Biol 1968, 21:325-331.

12. lida T, Yamamoto $\mathrm{K}$ : Cloning and expression of two genes encoding highly homologous hemolysins from a Kanagawa phenomenon-positive Vibrio parahaemolyticus T4750 strain. Gene 1990, 93:9-15.

13. Nishibuchi M, Kaper JB: Duplication and variation of the thermostable direct haemolysin $(t d h)$ gene in Vibrio parahaemolyticus. Mol Microbiol 1990, 4:87-99.

14. Park KS, Ono T, Rokuda M, Jang MH, Okada K, lida T, Honda T: Functional characterization of two type III secretion systems of Vibrio parahaemolyticus. Infect Immun 2004, 72:6659-6665.

15. Izutsu K, Kurokawa K, Tashiro K, Kuhara S, Hayashi T, Honda T, lida T: Comparative genomic analysis using microarray demonstrates strong correlation between presence of Vp-PAl and pathogenicity in Kanagawa phenomenon-positive Vibrio parahaemolyticus. Infect Immun 2008, 76:1016-1023.

16. Chatterjee S, Ghosh K, Raychoudhuri A, Chowdhury G, Bhattacharya MK, Mukhopadhyay AK, Ramamurthy T, Bhattacharya SK, Klose KE, Nandy RK: 
Incidence, virulence factors, and clonality among clinical strains of non01, non-0139 Vibrio cholerae isolates from hospitalized diarrheal patients in Kolkata, India. J Clin Microbiol 2009, 47:1087-1095.

17. Dziejman M, Serruto $D$, Tam VC, Sturtevant D, Diraphat $P$, Faruque $S M$, Rahman MH, Heidelberg JF, Decker J, Li L, Montgomery KT, Grills G, Kucherlapati R, Mekalanos JJ: Genomic characterization of non-O1, nonO139 Vibrio cholerae reveals genes for a type III secretion system. Proc Natl Acad Sci USA 2005, 102:3465-3470.

18. Henke JM, Bassler BL: Quorum sensing regulates type III secretion in Vibrio harveyi and Vibrio parahaemolyticus. I Bacteriol 2004, 186:3794-3805.

19. Murphy RA, Boyd EF: Three pathogenicity islands of Vibrio cholerae can excise from the chromosome and form circular intermediates. I Bacteriol 2008, 190:636-647.

20. Okada N, lida T, Park KS, Goto N, Yasunaga T, Hiyoshi H, Matsuda S, Kodama T, Honda T: Identification and characterization of a novel type III secretion system in trh-positive Vibrio parahaemolyticus strain TH3996 reveal genetic lineage and diversity of pathogenic machinery beyond the species level. Infect Immun 2009, 77:904-913.

21. lida T, Park KS, Honda T: Vibrio parahaemolyticus. In The Biology of Vibrios. Edited by: Thompson FL, Austin B, Swings J. Washington, DC: ASM Press; 2006:340-348

22. Kodama T, Rokuda M, Park KS, Cantarelli W, Matsuda S, lida T, Honda T: Identification and characterization of VopT, a novel ADPribosyltransferase effector protein secreted via the Vibrio parahaemolyticus type III secretion system 2. Cell Microbiol 2007, 9:2598-2609

23. Kodama T, Hiyoshi H, Gotoh K, Akeda Y, Matsuda S, Park KS, Cantarelli W, lida T, Honda T: Identification of two translocon proteins of Vibrio parahaemolyticus type III secretion system 2. Infect Immun 2008, 76:4282-4289

24. Livermans AD, Cheng HC, Trosky JE, Leung DW, Yarbrough ML, Burdette DL, Rosen MK, Orth K: Arp2/3-independent assembly of actin by Vibrio type III effector VopL. Proc Natl Acad Sci USA 2007, 104:17117-17122.

25. Vora GJ, Meador CE, Bird MM, Bopp CA, Andreadis JD, Stenger DA: Microarray-based detection of genetic heterogeneity, antimicrobial resistance, and the viable but nonculturable state in human pathogenic Vibrio spp. Proc Natl Acad Sci USA 2005, 102:19109-19114.

26. Li T, Kobayashi A, Takata N, Yoshimura T, Maehara Y, Tsuchiya T, Miyoshi S Role of the Enterotoxic Hemolysin in Pathogenicity of Vibrio mimicus. J Health Sci 2008, 54:686-691.

doi:10.1186/1471-2180-10-302

Cite this article as: Okada et al.: Presence of genes for type III secretion system 2 in Vibrio mimicus strains. BMC Microbiology 2010 10:302.

\section{Submit your next manuscript to BioMed Central and take full advantage of:}

- Convenient online submission

- Thorough peer review

- No space constraints or color figure charges

- Immediate publication on acceptance

- Inclusion in PubMed, CAS, Scopus and Google Scholar

- Research which is freely available for redistribution

Submit your manuscript at www.biomedcentral com/submit
Biomed Central 